\title{
Application of the Modified Cytosine Base-Editing in the Cultured Cells of Bama Minipig
}

\section{Jia-sheng Pan \\ Foshan University \\ Zi-sheng Lin \\ Foshan University \\ Jian-cong Wen \\ Foshan University \\ Jian-feng Guo \\ Foshan University \\ Xia-hui Wu \\ Foshan University \\ Ying-ying Liu \\ Foshan University \\ Wen-jun Lai \\ Foshan University \\ Qi-ying Liang \\ Foshan University \\ Yong-shi Xie \\ Foshan University \\ Yi-rou Chen \\ Foshan University \\ Yi-hong Chen \\ Foshan University \\ Ai-fen Yan \\ Foshan University \\ Juan Feng \\ Foshan University \\ Lian Liu \\ Foshan University}

\section{Dao-yuan Gong}

Foshan University

\section{Xiang-xing Zhu}

Foshan University https://orcid.org/0000-0002-5265-2620

Jia-hong Lu

University of Macau

Dong-sheng Tang ( $\nabla$ tangdsh@163.com )

Foshan University 


\section{Research Article}

Keywords: Genome-editing, Cytosine base editor, Myostatin, Genetic modification, Bama minipig

Posted Date: May 20th, 2021

DOI: https://doi.org/10.21203/rs.3.rs-473930/v1

License: (c) (i) This work is licensed under a Creative Commons Attribution 4.0 International License. Read Full License 


\section{Abstract}

Bama minipig is a unique miniature swine bred from China. Their favorable characteristics include delicious meat, strong adaptability, tolerance to rough feed, and high levels of stress tolerance. Unfavorable characteristics are their low lean meat percentage, high fat content, slow growth rate, and low feed conversion ratio. Genome-editing technology using CRISPR/Cas9 efficiently knocked out the myostatin gene (MSTM) that has a negative regulatory effect on muscle production, effectively promoting pig muscle growth and increasing lean meat percentage of the pigs. However, CRISPR/Cas9 genome editing technology is based on random mutations implemented by DNA double-strand breaks, which may trigger genomic off-target effects and chromosomal rearrangements. The application of CRISPR/Cas9 to improve economic traits in pigs has raised biosafety concerns. Base editor (BE) developed based on CRISPR/Cas9 such as cytosine base editor (CBE) effectively achieve targeted modification of a single base without relying on DNA double-strand breaks. Hence, the method has greater safety in the genetic improvement of pigs. The aim of the present study is to utilize a modified CBE to generate MSTN-knockout cells of Bama minipigs. Our results showed that the constructed "all-in-one"-modified CBE plasmid achieved directional conversion of a single C.G base pair to a T.A base pair of the MSTN target in Bama miniature pig fibroblast cells. We successfully constructed multiple single-cell colonies of Bama minipigs fibroblast cells carrying the MSTN premature termination and verified that there were no genomic off-target effects detected. This study provides a foundation for further application of somatic cell cloning to construct MSTN-edited Bama minipigs that carry only a single-base mutation and avoids biosafety risks to a large extent, thereby providing experience and a reference for the base editing of other genetic loci in Bama minipigs.

\section{Introduction}

Bama minipigs are a unique breed originating from Bama Yao Autonomous County in Guangxi, China. The breed has favorable characteristics including delicious meat, strong adaptability, tolerance to rough feed, and a high level of stress tolerance. Bama minipigs have played an important role in local economic growth and agriculture, and thereby in poverty reduction. In addition to food consumption, because of its small size, Bama minipigs have become a well-known local specialty throughout the country, with a high value in the area's economic development (Zhu et al., 2020a; b). Characteristic traits of the breed include early sexual maturity, large litters, and a high degree of inbreeding. The pigs also have the potential to be used as medical disease models (Zhu et al., 2018). However, similar to other indigenous pig breeds in China, Bama minipigs have unfavorable characteristics such as low lean meat percentage, high fat content, slow growth rate, and low feed conversion ratio, hindering the breed's application and promotion (Zhu et al., 2020a; b).

Traditional genetic selection in pigs involves long breeding cycles, high costs, and limited genetic resources, particularly regarding increased yield, improvements in quality, or enhancement of stress tolerance. In contrast, genome-editing technology represented by CRISPR/Cas9 effectively modifies the genome of pigs using modified cells carrying the target genes as nuclear donors for somatic cell cloning; this method can achieve stable improvement of one or more production traits (Petersen et al., 2017; Ruan et al., 2017). Hence, the technology is expected to have significant potential for genetic improvement of pigs. By generating a double-strand break at the target genetic locus, CRISPR/Cas9 genome editing technology relies on the DNA repair mechanism of the cells to introduce a certain number of base insertions or deletions at the break sites, efficiently disrupting gene expression (Zhao et al., 2019). However, the editing results triggered by CRISPR/Cas9 are random and rely on DNA doublestrand breaks; this may cause genomic off-target effects, deletion of large gene fragments, abnormal chromosomal structure, and even whole chromosome deletions, leading to unpredictable results in the edited cells (Kosicki et al., 
2018; Zuccaro et al., 2020). Because of these shortcomings, the application of CRISPR/Cas9 to improve economic traits in pigs has led to biosafety concerns.

To ensure a high precision of genome editing, base editor (BE) technology that achieves single-base targeted mutations has emerged. In 2016, Liu and colleagues fused the Cas9n protein D10A (which only leads to DNA singlestrange cleavage) with rat cytosine deaminase, rAPOBEC (which catalyzes the deamination of $C$ into $U$, and $U$ is recognized as T during DNA replication), and uracil DNA glycosylase inhibitor, UGI (which prevents uracil glycosylase from glycosylation of $U$ to cause base excision repair) (Komor et al., 2016). This fusion protein specifically targets $C \cdot G$ base pairs for mutation to $T \cdot A$ base pairs under the guidance of single-stranded guide RNA (sgRNA); the protein is also known as cytosine base editor (CBE). CBE does not produce DNA double-strand breaks, rather only resulting in the targeted mutation of a single $C \cdot G$ base pair to a $T \cdot A$ base pair, and thus is more precise than the original CRISPR/Cas9 genome editing technology (Komor et al., 2016). CBE is expected to be safer in genetic improvement of pigs. To date, the application of CBE in genetic modification of pigs has achieved several breakthroughs. Although BEs have been successfully used to produce genetically engineered pigs (Li et al., 2018; Xie et al., 2019; Wang et al., 2020), the BEs used in the past, such as BE3, were shown to cause a high proportion of DNA and RNA off-target effects (Komor et al., 2016). Improved versions of BE (e.g., YE1-BE4maxNG) with the systematic transformation through protein engineering technology have been constructed; these have a lower level of RNA editing activity and fewer insertion/deletion mutations while maintaining the efficiency of base editing (Doman et al., 2020). These improved versions of BEs will become increasingly important in the genetic improvement of pigs.

The most significant unfavorable characteristics of Bama minipigs are the low lean meat percentage and high fat content. Our research team particularly aims to utilize genome editing technology to increase the lean-meat yield of Bama minipigs by essentially eliminating the key genes that inhibit the muscle growth of the animals. Myostatin gene (MSTM), also known as growth differentiation factor 8 (GDF8), is mainly expressed in skeletal muscle and has a negative regulatory effect on muscle development and regeneration. The well-known double-muscled hips in Belgian Blue Cattle is a significant developmental phenotype produced by a loss-of-function mutation in MSTN (Grobet et al., 1997). Studies have shown that MSTN-knockout mediated by zinc-finger nucleases (ZFNs), transcription activator-like effector nucleases (TALENs) and CRISPR/Cas9 replicate the phenotype of doublemuscled hips in rabbits, cows, goats, sheep, and pigs, thereby significantly increasing the lean meat percentage and reducing the fat content of the animals (He et al., 2018; Lv et al. 2016; Wang et al., 2016). Thus, MSTNis an ideal target for preparing Bama minipigs with significantly increased lean meat percentage.

In view of the fact that base editing has more precise genome editing effects and greater safety in the genetic improvement of livestock, this study aimed to use modified CBE to prepare MSTN-knockout cells of Bama minipigs by firstly constructing an "all-in-one" modified CBE plasmid and verifying that this plasmid achieved the directional conversion of a single C.G base in the editing window to T.A base pairs at multiple gene loci in the cells of Bama minipigs. Subsequently, four potential targets (CBE introduces premature stop codons to disrupt MSTN function) were designed according to the characteristics of the editing window of CBE, followed by comparing their activities to select the most efficient target among them for the preparation of single-cell colonies. Lastly, the prepared singlecell colonies and base-editing effects were identified, and the genomic off-target effects on the single-cell colonies with expected base edits were analyzed. We constructed and used the modified CBE plasmid to successfully prepare single-cell colonies of Bama minipigs carrying the MSTN premature termination and without genomic offtarget effects. This study provides a foundation for further application of somatic cell cloning to construct MSTN- 
edited Bama minipigs that carry only a single-base mutation and avoids biosafety risks to a large extent, thereby providing a reference for the base editing of other genetic loci in Bama minipigs.

\section{Materials And Methods 2.1 Animal ethics}

All of the animal procedures used in this study were carried out in accordance with the Guide for Care and Use of Laboratory Animals (8th edition, released by the National Research Council, USA), and were approved by the Animal Care \& Welfare Committee of Foshan University (approval no. 2019020). All of the surgical procedures were performed under anesthesia by a veterinarian, and all efforts were made to minimize animal suffering.

\subsection{Reagents and chemicals}

All of the reagents used in this study were purchased from Sigma-Aldrich Company (St. Louis, MO, USA) unless stated otherwise. Milli-Q ultrapure water (Millipore, Bedford, MA, USA) was used for the preparation of solutions. Self-made solutions were filtered through a $0.22-\mu \mathrm{m}$ filter (Millipore) and stored at $4^{\circ} \mathrm{C}$ or at $-20^{\circ} \mathrm{C}$ until use. Pipette tips, centrifuge tubes, and petri dishes were purchased in aseptic packages and were all disposable.

\subsection{Preparation of "all-in-one" modified modified CBE vector}

The sequence information of the modified CBE vector (namely as YE1-BE4maxNG) created by Doman et al. (2020) was obtained from the Addgene (catalog \#138159). The sequence was synthesis and cloned into the pUC cloning vector (BGI, Shenzhen, China). For construction of CBE vector, the YE1-BE4maxNG fragment with Agel/Sall restriction sites was obtained by PCR, and linked to the PX459 vector by molecular cloning. The accuracy of molecular cloning was tested by gene sequencing, and the obtained plasmid was named as PX-YE1-BE4maxNG. Complete sequence information of PX-YE1-BE4maxNG were provided online. The gRNA and CBE expression elements were integrated into one vector, namely as "all-in-one" vector. The "all-in-one" vector is very suitable for cell transfection.

\subsection{Construction of CBE plasmid for transfection}

Design and construction of the CBE plasmid were performed according to our previous studies (Wei et al., 2020; Zhu et al., 2020a; b). The sgRNAs used in this study were designed as described below, and were produced by BGI company. PX-YE1-BE4maxNG vector was linearize using Bsbl restriction-enzyme digestion, and linked with the annealed sgRNAs using a T4 DNA Ligase (TaKaRa, Dalian, China). The DNA linking products were transduced into competent Escherichia coli. cells, were seeded into LB medium (supplemented with $50 \mu \mathrm{g} / \mathrm{ml}$ of ampicillin) and cultured overnight with shaking at $37^{\circ} \mathrm{C}$. We confirmed the constructed CBE base-editing plasmid by DNA sequencing (BGI). Plasmids were purified using an EndoFree Maxi Plasmid Kit (Tiangen, Beijing, China) according to the manufacturer's instructions, and purified plasmids were then stored at $-20^{\circ} \mathrm{C}$ for future use. The DNA concentration was estimated using a NanoDrop spectrophotometer (NanoDrop Technologies Inc., Washington, USA).

\subsection{Preparation of Bama minipig fibroblast cells}

Procedures used for the isolation, cultivation, and transfection of kidney fibroblasts from newborn Bama minipigs were based upon our previous studies (Wei et al., 2020; Zhu et al., 2020a; b). After deep anesthetization, the newborn piglet was euthanized and both kidneys were removed and minced in Dulbecco's phosphate-buffered saline (DPBS; 
Gibco, Grand Island, NY, USA). Tissue fragments were washed several times with DPBS and digested in a $0.25 \%$ $(\mathrm{w} / \mathrm{v})$ trypsin-EDTA solution for 30 minutes at $37^{\circ} \mathrm{C}$. Isolated cells were cultured for 1 to 2 passages in Dulbecco's modified Eagle's medium (DMEM; Gibco) supplemented with 20\% (v/v) fetal bovine serum (FBS; Gibco) and then frozen in liquid nitrogen for future use.

\subsection{Activity test of base-editing in Bama minipig cells}

In human cells, previous studies have shown that the editing window spanning of modified CBE vector (YE1BE4maxNG) was located at positions 3 to 9 (counting the protospaceradjacent motif (PAM) as positions 21 to 23 ). To assess the base-editing activity of the modified CBE editor on Bama minipig cells, we selected several genomeediting sites, including sites of genes related to economic trait locus (CD163, MSTN site 1) and those of genes related to human disease, apolipoprotein A5 (APOA5) and the low-density lipoprotein receptor ( $L D L R)$, which are being used for gene editing using CRISPR/Cas9 system in our team. All the gene loci contained the expected CBEediting sites. The sgRNAs for the tested gene sites were listed in Table 1.

Table 1

Target sites (sgRNAs) and primers used for activity test of base-editing in Bama minipig cells

\begin{tabular}{|c|c|c|c|c|}
\hline No. & Gene* & Target sequences (sgRNAs)** & Primers & Amplicon size (bp) \\
\hline \multirow[t]{2}{*}{1} & Apoa 5 & agccagagcagcgggaacaaagg & PF: Tcagagcacatcatggcaag & 539 \\
\hline & & & PR: cattctgaaggctgcagttg & \\
\hline \multirow[t]{2}{*}{3} & $L D L R$ & cctgcatccctgagctgtgggcc & PF: Ctcactgggcttggcctg & 489 \\
\hline & & & PR: agttctcctcgtcagacttg & \\
\hline \multirow[t]{2}{*}{2} & CD163 & tttcagcccacaggaaacccagg & PF: Aggtctagaatcggctaagc & 536 \\
\hline & & & PR: gatgtctgtgactacctaac & \\
\hline \multirow[t]{2}{*}{4} & MSTN site 1 & ccaggagaagatgggctggtaag & PF: Gctgatcttctaatgcaagtg & 545 \\
\hline & & & PR: cataggatatgaaactgaacac & \\
\hline & $\begin{array}{l}\text { Apoa5, ger } \\
\text { yene for co } \\
\text { ositions } 3\end{array}$ & $\begin{array}{l}\text { coding apolipoprotein A-V; } L \\
\text { CD163 molecule; } M S T N \text {, gen } \\
\text { are shown in red. The protos }\end{array}$ & $\begin{array}{l}\text { yene for coding low density li } \\
\text { oding myostatin. } \\
\text { adjacedicedicted } \\
\text { adiacent (PAM) sequen }\end{array}$ & $\begin{array}{l}\text { otein receptor; } \\
\text { e editor targeting } \\
\text { are marked in bold. }\end{array}$ \\
\hline
\end{tabular}

For identification of the CBE-mediated base-editing in cultured cells, 100,000 Bama minipig kidney fibroblast cells in passage number 1 to 2 were transfected using Lipofectamine 3000 according to the manufacturer's instruction. One day later, the cells were split into 35-mm cell-culture dishes (NUNC). After $24 \mathrm{~h}$ of recovery, the transfected cells were selected with $1.0 \mu \mathrm{g} / \mathrm{mL}$ puromycin (Solarbio, Beijing, China) for 3 days; and after puromycin was withdrawn, we continuously cultured the cells for 5 to 7 days. For each test of these gene loci, three independent cell transfection were performed. The cells from three independent cell transfection were pooled and genomic DNA was extracted by using a TIANamp Genomic DNA Kit (Tiangen, Beijing, China).

Primers were designed to amplify across the gene-editing sites (Table 1). PCR reactions were conducted with $2 \mu \mathrm{L}$ of genomic DNA, $0.5 \mu \mathrm{L}$ of forward primer $(10 \mu \mathrm{M}), 0.5 \mu \mathrm{L}$ of reverse primer $(10 \mu \mathrm{M})$, and $10 \mu \mathrm{L}$ of PrimeSTAR Max (TaKaRa, Dalian, China); and we then added deionized water to a total volume of $20 \mu \mathrm{L}$. PCR amplification conditions were as follows: 1 cycle at $95^{\circ} \mathrm{C}$ for $5 \mathrm{~min} ; 35$ cycles at $98^{\circ} \mathrm{C}$ for $10 \mathrm{sec}, 56^{\circ} \mathrm{C}$ for $5 \mathrm{sec}$, and $72^{\circ} \mathrm{C}$ for 5 
sec; followed by $72^{\circ} \mathrm{C}$ for 5 min. We examined the PCR products using $1.5 \%(\mathrm{w} / \mathrm{v})$ agarose gel electrophoresis containing $0.01 \%(\mathrm{v} / \mathrm{v})$ Andy Gold ${ }^{\mathrm{TM}}$ Nucleic Acid Gel Stain (Applied BioProbes, Davis, CA, USA).

PCR amplicons were purified using universal DNA purification kit (Tiangen) and sent to the GENEWIZ company for Sanger and next generation sequencing. For next generation sequencing, amplicons were ligated to adapters and sequencing was performed on an Illumina MiSeq 2x300bp sequencing platform. For each test, more than $50 \mathrm{k}$ reads were collected. The CBE-mediated base-editing was determined by aligning the reads to the wild type sequences.

\subsection{Generation of Bama minipig cell colonies harboring CBE- mediated MSTN knock-out}

The modified "all-in-one" CBE vector was employed for generation of Bama minipig cell colonies harboring CBEmediated MSTN knock-out. Firstly, four potential targets (MSTN sites $2 \sim 5$ ) (Table 2) which can introduces premature stop codons (TAG, TGA, or TAA) to disrupt MSTN function by using CBE-mediated C-to-T transformation have been tested as described above. Then, the target with the highest CBE-mediated base-editing activity was used for generation of Bama minipig cell colonies harboring CBE-mediated MSTNknock-out.

Table 2

Target sites (sgRNAs) and primers used for activity test of CBE-mediated MSTN disrupution in Bama minipig cells

\begin{tabular}{|c|c|c|c|c|}
\hline No. & Gene* & Target sequences (sgRNAs)** & Amplicon size (bp) & Amplicon size (bp) \\
\hline \multirow[t]{2}{*}{1} & MSTN site 2 & cagcgagcaaaaggaaaatgTGG & PF: Aacctctgacagcgagattc & 524 \\
\hline & & & PR: tggacatcgtactgatcaatc & \\
\hline \multirow[t]{2}{*}{2} & MSTN site 3 & aaacaacctgaatccaacttAGG & PF: Gctgatcttctaatgcaagtg & 545 \\
\hline & & & PR: cataggatatgaaactgaacac & \\
\hline \multirow[t]{2}{*}{3} & MSTN site 4 & CCAGGCACTGGTATTTGGCAGAG & PF: Gctgatcttctaatgcaagtg & 545 \\
\hline & & & PR: cataggatatgaaactgaacac & \\
\hline \multirow[t]{2}{*}{4} & MSTN site 5 & CCCAGGCACTGGTATTTGGCAGA & PF: Gctgatcttctaatgcaagtg & 545 \\
\hline & & & PR: cataggatatgaaactgaacac & \\
\hline
\end{tabular}

For generation of Bama minipig cell colonies, the CBE plasmid-transfected cells were subcultured in 60-mm culture dishes. After $48 \mathrm{~h}$, the cells were treated with $1.0 \mu \mathrm{g} / \mathrm{ml}$ puromycin for three days to eliminate the non-transfected cells. After removing drug-resistant cells, the remaining cells were further cultured for nine to ten days. Once a single-cell clone with a diameter of 2-3 mm (approximately 1,000 cells) was found, the single-cell colony was isolated and inoculated into a 4-well culture clusters (NUNC) for further incubation to confluence. The cell colonies were subcultured and part of them was collected for base-editing identification. Positive base-editing cell colonies were expanded and then cryopreserved. PCR amplicons produced as described above were sent out for Sanger DNA sequencing. The CBE-mediated base-editing was determined by aligning the reads to the wild type sequences.

\subsection{Genomic off-target detection in base-edited cell colonies}


Potential OTSs were predicted by the CRISPR Design Tool (http://crispor.tefor.net) according to our previous studies (Wei et al., 2020; Zhu et al., 2020a; b). Six sites with potential genomic off-target effects for MSTNlocus were selected for detection of off-targets occurred in base-edited cell colonies (Table 3). Specific primers were used for $\mathrm{PCR}$ reaction, and the products were sequenced to confirm whether genomic off-targeting mutations existed. Genomic off-targets were identified by alignment of sequenced alleles to wild type allele.

Table 3

Off-target sites (OTS) and corresponding primers used for analysis of CBE-mediated base-editing in MSTN site5 in Bama minipig cells

\begin{tabular}{|c|c|c|c|c|c|}
\hline $\begin{array}{l}\text { Off- } \\
\text { target } \\
\text { No. }\end{array}$ & Chr. & Sequence* & Score & $\begin{array}{l}\text { Primers for PCR and } \\
\text { sequencing }\end{array}$ & $\begin{array}{l}\text { Amplicon } \\
\text { (bp) }\end{array}$ \\
\hline \multirow[t]{2}{*}{$\begin{array}{l}\text { MSTN } \\
\text { site } 5\end{array}$} & Chr15 & TCTGCCAAATACCAGTGCCTGGG & 100 & $\begin{array}{l}\text { F: } \\
\text { GCTGATCTTCTAATGCAAGTG }\end{array}$ & 545 \\
\hline & & & & $\begin{array}{l}\text { R: } \\
\text { CATAGGATATGAAACTGAACAC }\end{array}$ & \\
\hline \multirow[t]{2}{*}{$\begin{array}{l}\text { Off- } \\
\text { target } 1 \#\end{array}$} & Chr2 & TCTGCCAAATACCAGTGCTTGAG & 6.3 & $\begin{array}{l}\text { F: } \\
\text { CAGTTTAGGAGAGAGGGAAG }\end{array}$ & 457 \\
\hline & & & & $\begin{array}{l}\text { R: } \\
\text { ATGTGGTAGTCACAGAGCTC }\end{array}$ & \\
\hline \multirow[t]{2}{*}{$\begin{array}{l}\text { Off- } \\
\text { target 2\# }\end{array}$} & Chr7 & TCTTCCAAATGCCAGTGCCTGGG & 3.93 & $\begin{array}{l}\text { F: } \\
\text { GTCTGGGATCTTCAGCAAAG }\end{array}$ & 700 \\
\hline & & & & $\begin{array}{l}\text { R: } \\
\text { TATTGTGTCACACACGGGTC }\end{array}$ & \\
\hline \multirow[t]{2}{*}{$\begin{array}{l}\text { Off- } \\
\text { target 3\# }\end{array}$} & Chr12 & СCTCCCAAACACCAGTGCCTGGG & 2.46 & $\begin{array}{l}\text { F: } \\
\text { ATCAGACCTAGGAGACCAAG }\end{array}$ & 548 \\
\hline & & & & $\begin{array}{l}\text { R: } \\
\text { GGATTGTGGCATAGGCTTTG }\end{array}$ & \\
\hline \multirow[t]{2}{*}{$\begin{array}{l}\text { Off- } \\
\text { target } 4 \#\end{array}$} & Chr3 & TATTACAAATACCAGTGCCTAGG & 2.32 & $\begin{array}{l}\text { F: } \\
\text { TTTGCCATTTCTTGGGCCAC }\end{array}$ & 456 \\
\hline & & & & R: ATGTCCTTTCTCСTCATCCC & \\
\hline \multirow{2}{*}{$\begin{array}{l}\text { Off- } \\
\text { target } 5 \#\end{array}$} & Chr5 & GATGCCCAATACCAGTGCCTGGG & 1.74 & F: TAGTCCTGCTACCATGCATC & 668 \\
\hline & & & & $\begin{array}{l}\text { R: } \\
\text { TGGGCTGTATCTTCCAGAAG }\end{array}$ & \\
\hline \multirow[t]{2}{*}{$\begin{array}{l}\text { Off- } \\
\text { target } 6 \#\end{array}$} & Chr2 & СCTGCCAAAAACCAGTGCCTGGA & 1.48 & $\begin{array}{l}\text { F: } \\
\text { TGTTACTGCTGAGCCACAAC }\end{array}$ & 502 \\
\hline & & & & $\begin{array}{l}\text { R: } \\
\text { AACTGCGTGACAGTGTTAGG }\end{array}$ & \\
\hline
\end{tabular}

${ }^{*}$ Red letters are the mismatched nucleotides in off-target sequences aligned to sgRNA. PAM sequences are underlined.

\section{Results}




\subsection{Construction of the modified CBE vector and detection of the base- editing activity}

We first obtained the modified CBE vector sequences through gene synthesis (YE1-BE4maxNG) and then linked them to the conventional PX459 vector by molecular cloning. The accuracy of molecular cloning was assessed by gene sequencing, and the plasmids so produced were named PX-YE1-BE4maxNG. The complete sequence information of PX-YE1-BE4maxNG were provided online.. The gRNA and CBE expression elements were integrated into one vector, namely as "all-in-one" vector (Fig. 1A). The "all-in-one" vector is very suitable for cell transfection.

To test the activity of the constructed "all-in-one" modified CBE plasmid on the cells of Bama minipigs, four endogenous genetic loci were selected for activity testing (these loci were used as CRISPR/Cas9 genome editing targets for gene knockout experiments in our team using the cells of Bama minipigs), the target genes being APOA5 and the LDLR for the construction of human disease models and the CD163 and MSTN(site 1) genes for the improvement of economic traits in pigs (see Fig. 1B for the specific information concerning the loci). After cell transfection, puromycin screening, the cells from three independent cell transfection were pooled and genomic DNA was extracted for PCR. The PCR products were subjected to Sanger sequencing and second-generation sequencing.

Our results showed that the conversion efficiencies of C-to-T bases of the four loci of APOA5, LDLR, CD163, and MSTN(site 1) were $8.7 \%, 30.1 \%, 7.4 \%$, and $10.3 \%$, respectively, indicating that the bases of CBE plasmids constructed in this study exerted editing activity and that the editing efficiency varied due to differences in the edited loci (Fig. 1B,C).

The editing results of the CBE were subsequently analyzed in the editing window. The results indicated that the editing window of CBE was the 3rd to 7th positions of gRNA, with the 5th and 6th positions showing the highest efficiency (counting the protospaceradjacent motif (PAM) as positions 21 to 23) (Fig. 1B,C). These results indicated that the "all-in-one" modified CBE plasmid constructed in this study achieved single C.G base pair to T.A base pair directional conversion in the cells of Bama minipigs.

\subsection{Target design for CBE-mediated introduction of a premature stop codon into MSTN in Bama minipig cells and activity test}

MSTN negatively regulates muscle development and regeneration. MSTNknockout is normally achieved by CRISPR/Cas9-mediated random base insertion/deletion leading to frameshift mutations. In this study, based on the directional conversion of single $C \cdot G$ base pairs to $T \cdot A$ base pairs mediated by CBE, the MSTN function was destroyed by introducing a premature stop codon, TAG, TGA, or TAA, into the MSTN coding frame. The MSTN of Bama minipigs consists of three exons. Existing studies have shown that the functional activity domain of MSTN is in exon 3. Thus, the exons 1 and 2 were used as the targets of gene editing in this study (Fig. 2A).

According to the characteristics of the CBE editing window revealed by the above experiments (i.e., positions 3 to 7 of gRNA, among which positions 5 and 6 had the highest editing efficiency), the target design of MSTN exons 1 and 2 of Bama minipigs was performed comprising four sgRNAs (namely sgRNA1, sgRNA2, sgRNA3, and sgRNA4) (Fig. 2A; the loci information were presented in Table 2). These four sgRNAs were constructed into separate CBE vectors and subsequently transfected into the cells of Bama minipigs to test for editing activity. After PCR and Sanger DNA sequencing, the results showed that sgRNA4 produced the highest percentage of targeted mutations (29.8\%; Fig. 2A,B). Thus, sgRNA4 was used to prepare CBE-mediated MSTN base-editing cell colonies. 


\subsection{Preparation of Bama minipig cell colonies harboring CBE-mediated MSTN knock-out}

The sgRNA4 with the best base-editing activity was used to prepare CBE-mediated MSTNknock-out Bama minipig cells in this study. Three cell transfections were performed successively in this study, and a total of 28 single-cell colonies were collected. Twenty-one out of the 28 single-cell colonies were cultured successfully. As shown in Table 4 and Fig. 3, gene sequencing showed that six single-cell colonies $(28.6 \%, 6 / 21)$ had the expected CBEmediated C-to-T transformation which could introduce a premature stop codon (TAG, TGA, or TAA) for disruption of the MSTN function. Of these, two $(9.5 \%, 2 / 21)$ were biallelic mutations.

Table 4

Summary of MSTN site 5 genotypes of Bama minipig single-cell colonies generated by CBE-mediated base-editing

\begin{tabular}{|c|c|c|}
\hline $\begin{array}{l}\text { Colony } \\
\text { No. }\end{array}$ & MSTN site5 genotypes* & Summary** \\
\hline $\begin{array}{l}\text { WT } \\
\text { control }\end{array}$ & TCTCTGAAACTTGACATGAACCCAGGCACTGGTATTTGGCAGAGCATTGATGTGAAGACA & WT \\
\hline \multirow[t]{2}{*}{$1-1 \#$} & TCTCTGAAACTTGACATGAACCCAGGCACTGGTATTTGGCAGAGCATTGATGTGAAGACA & WT \\
\hline & TCTCTGAAACTTGACATGAACCCAGGCACTGGTATTTAACAGAGCATTGATGTGAAGACA & Converted \\
\hline \multirow[t]{2}{*}{$1-4 \#$} & TCTCTGAAACTTGACATGAACCCAGGCACTGGTATTTGGCAGAGCATTGATGTGAAGACA & WT \\
\hline & TCTCTGAAACTTGACATGAACCCAGGCACTGGTATTTAACAGAGCATTGATGTGAAGACA & Converted \\
\hline \multirow[t]{2}{*}{$1-6 \#$} & TCTCTGAAACTTGACATGAACCCAGGCACTGGTATTTGGCAGAGCATTGATGTGAAGACA & WT \\
\hline & TCTCTGAAACTTGACATGAACCCAGGCACTGGTATTTAACAGAGCATTGATGTGAAGACA & Converted \\
\hline \multirow[t]{2}{*}{$1-7 \#$} & TCTCTGAAACTTGACATGAACCCAGGCACTGGTATTTGGCAGAGCATTGATGTGAAGACA & WT \\
\hline & TCTCTGAAACTTGACATGAACCCAGGCACTGGTATTTAACAGAGCATTGATGTGAAGACA & Converted \\
\hline $2-1 \#$ & TCTCTGAAACTTGACATGAACCCAGGCACTGGTATTTAACAGAGCATTGATGTGAAGACA & Converted \\
\hline $2-11 \#$ & TCTCTGAAACTTGACATGAACCCAGGCACTGGTATTTAACAGAGCATTGATGTGAAGACA & Converted \\
\hline \multicolumn{3}{|c|}{$\begin{array}{l}\text { Notes: *The sgRNA5 sequences are underlined, protospacer adjacent motif (PAM) sequences are labeled with } \\
\text { bold fonts. CBE-mediated conversion of C-to-T (in the complementary chain is G-to-A) are marked in red. **WT, } \\
\text { wild type. }\end{array}$} \\
\hline
\end{tabular}

\subsection{Genomic off-target identification}

Given its inability to produce DNA double-strand breaks, CBE (especially the modified CBE) is considered to have higher gene editing precision than CRISPR/Cas9. To verify the genomic off-target effect of modified CBE on the cells of Bama minipigs, we used four single-cell colonies with good growth activity and cell morphology and carrying target gene mutations for off-target effect detection. Six genomic off-target sites with high off-target scores (Score >1) were screened by the CRISPR Design Tool (http://crispor.tefor.net) (Table 3), followed by performing PCR on four single-cell colonie genomes independently and subsequent Sanger DNA sequencing. The results showed no conversion of C.G base pairs to T.A base pairs at the six selected off-target sites and no base deletion/insertion mutations caused by DNA double-strand breaks detected in the four single-cell colonies (Fig. 4), indicating that the 
modified CBE did not cause detectable genomic off-target mutations in the targets selected in this study and that the method had high genome-editing precision.

\section{Discussion}

\subsection{Gene editing offers great application potential in genetic improvement of pigs}

Traditional molecular breeding is still the main method for the genetic improvement of livestock. However, genomic editing technology is a developing trend due to its favorable characteristics of high efficiency and low cost (Petersen et al., 2017; Ruan et al., 2017; Zhao et al., 2019). To date, CRISPR/Cas9 genome technology has produced a number successful results in the genetic improvement of pigs. For example, Burkard and colleagues used CRISPR/Cas 9 technology to knock out the cell receptor CD163 of porcine reproductive and respiratory syndrome virus invading the pig body and successfully obtained gene-edited pigs that resisted the infection of reproductive and respiratory syndrome virus (Burkard et al., 2017). In 2018, and colleagues used CRISPR/Cas9-mediated gene knock-in technology to successfully obtain gene-edited pigs expressing interference RNA resistant to the classical swine fever virus (Ding et al., 2018). The in vivo and in vitro challenges demonstrated that the gene-edited pigs effectively resisted the infection of swine fever virus and had significantly reduced the clinical symptoms caused by swine fever virus as well as reduced mortality. In 2020, Xu et al. used CRISPR/Cas9 to construct CD163-and phosphorylated aminopeptidase $\mathrm{N}(p A P N)$-double knockout pigs, showing that multi-gene editing pigs resisted multiple viruses (Xu et al., 2020a). Recently, Han et al. used CRISPR/Cas9-mediated homologous recombination to successfully construct a gene-edited large white pig by specifically knocking out the lactoferrin gene at the 3' end of the alpha-s1 casein gene, which increased the lactoferrin content in sow milk and improved the survival rate of the piglets (Han et al., 2020). The above studies have indicated that CRISPR/Cas9 genome editing technology has extremely high application value in the genetic improvement of pigs.

The pig breeding program in China has long focused on genetic selection to reduce fat deposits and increase lean meat percentage. In the past few decades, genes related to economic traits such as growth promotion, muscle production, and fat consumption have been identified to improve economically important traits through genome editing. MSTNis a convenient target encoded by the GDF8 locus, as the protein is mainly expressed in skeletal muscle and has a negative regulatory effect on muscle development and regeneration (McPherron and Lee, 1997). Given the function of this gene in the growth and development of skeletal muscle, GDF8 has become an important target in genome editing technology for livestock to increase the lean meat percentage, reduce the fat content, and improve the meat quality. Qian et al. successfully obtained MSTN mutant gene-edited Meishan pigs using ZFN technology and showed that the MSTN mutant pigs had more lean meat and less body fat, traits that significantly improved meat production (Qian et al., 2015). Subsequently, Rao et al. used TALEN technology to successfully obtain MSTN gene-edited pigs and showed that the muscle fibers of these pigs became thinner and more numerous, exhibiting the double-muscled phenotype in the hips (Rao et al., 2016). In 2020, Li et al. and our group obtained MSTNknockout miniature pigs and Bama minipigs, respectively (Li et al., 2020; Zhu et al., 2020b). Both breeds showed an increase in the number of muscle fibers and a decrease in muscle fiber size, indicating that the geneediting mediated MSTN-knockout significantly improved the meat production traits of pigs and increased their economic value. 


\subsection{Modifield CBE offers a safer strategy for genomic-editing}

Although the CRISPR/Cas9 technology has shown great potential in the genetic breeding of pigs, with the deepening of research, the safety risks of CRISPR/Cas9 have aroused increasing attention. Since 2018, many studies have shown that the CRISPR system activates p53 to cause DNA damage and ultimately interferes with its gene editing efficiency (Haapaniemi et al., 2018; Ihry et al. 2018). In 2018, Kosicki et al. reported that the repair of double-strand breaks caused by CRISPR/Cas9 genome editing tools in mouse embryonic stem cells, mouse hematopoietic progenitor cells, and human differentiated cell lines causes the loss of large gene fragments, resulting in pathogenic consequences (Kosicki et al., 2018). In 2020, Zuccaro et al. showed that many DNA double-strand breaks induced by CRISPR/Cas9 gene editing in human early embryos could not be repaired, eventually leading to the loss of large chromosomal fragments and even loss of entire chromosomes (Zuccaro et al., 2020). In the same year, Xu et al. showed that in the absence of exogenous sgRNAs, the Cas9 protein still directly bound to the KU86 subunit in the DNA-dependent protein kinase (DNA-PK) complex, thereby inhibiting the DNA-PK complex-dependent nonhomologous end joining pathway to cause DNA damage (Xu et al., 2020b). These risks inevitably hinder the application of CRISPR/Cas9 genome editing technology in the genetic improvement of pigs.

In 2016, Komor et al. reported a tool for genome editing that did not require double-stranded DNA cleavage or a donor template, i.e., a base editing tool, that avoided the double-stranded DNA breaks generated by the CRISPR/Cas 9 genome editing and did not rely on the donor template, thereby achieving the directional conversion of a single $C \cdot G$ base pair to a $T \cdot A$ base pair in the editing window with the characteristics of higher precision and greater safety (Komor et al., 2016). Single-base editing does not produce double-strand breaks, avoiding the risk of DNA damage and chromosome fragmentation in cells. Many laboratories are currently conducting research on gene editing in pigs mediated by base editing tools. For example, Li et al. used BE3 to construct twist-related protein 2 gene (TWIST2) and tyrosinase gene (TYR)-edited porcine fibroblasts and produced TWIST2-and TYR-edited pigs by somatic cell nuclear transfer to construct pig models of congenital eyelid defects, ablepharon-macrostomia syndrome (AMS), and albinism (Li et al., 2018). Xie et al. used BE3 or hA3A-BE3 combined with embryo injection and somatic cell cloning to construct Duchenne muscular dystrophy gene-edited pigs, lamin A/C gene-edited pigs, and $R A G 1, R A G 2$, IL2RG multi-gene edited pigs (Xie et al., 2019). Wang et al. combined the Streptococcus pyogenes Cas9-NG mutant with hA3A-BE3 to construct the hA3A-BE3-NG system and performed C-to-T transformation at the sites of NGN protospacer adjacent motif, resulting in the production of multiple economic trait-related genes (CD163, APN, MSTN, and melanocortin 4 receptor genes) in porcine fibroblasts (Wang et al., 2020). Nonsense and missense mutations have great potential in the improvement of animal genetics and breeding. Yuan et al. used AncBE4max to perform multi-gene editing of GGTA1, B4galNT2, and CMAH pigs that could be used as organ donors for xenotransplantation (Yuan et al., 2020). These results indicated that base editing tools provide a new option for genetic improvement of pigs.

However, the initial version of CBE also had significant shortcomings. In 2019, Zuo et al. reported that the CBE editing tool had many off-target edits in mouse embryos (Zuo et al., 2019). The application of CBE editing tools on plants also showed similar effects. For example, Jin et al. used BE3, HF-BE3, and ABE to treat rice plants and used whole-genome sequencing analysis to show that BE3 and HF-BE3 (but not ABE) induced many whole-genome offtarget mutations (Jin et al., 2019). Most of the mutations were C-to-T single nucleotide variants. With the benefits of the optimization of protein engineering, researchers have optimized CBE to reduce its off-target activities. Doman et al. reported an improved version of the CBE tool, Y1-BE4max-NG, that minimized DNA off-target effects while maintaining the original target base editing activity (Doman et al., 2020). Such modifield CBE offers a safer strategy for genomic-editing.

Page $12 / 21$ 


\subsection{Modified CBE vector provided a simple, safe and effective base-editing in Bama minipig cells}

The goal of the authors' team is to use gene editing techniques efficiently and safely to improve the production traits of Bama minipigs. We believe that the improved version of CBE provided higher precision and safety for the genetic improvement of pigs. The improved version of the CBE tool, Y1-BE4max-NG, was used in this study. To facilitate the application to porcine cells, this study integrated the improved version of CBE tool and gRNA expression elements into the same plasmid. To improve the effect of the CBE tool on pigs, we also tested four genetic loci in Bama minipigs. Our results showed that the editing efficiency ranged from $8.7-30.1 \%$, varying between different genetic loci. The editing window was concentrated in 4-7 positions, with the most efficient editing sites in positions 5 and 6, similar to the findings of Doman et al. on the engineered cells (e.g., 293T) (Doman et al., 2020). However, compared with the engineered cells, the overall editing efficiency of the improved CBE tool in the porcine fibroblasts was lower, possibly due to the different cell types and editing targets. Although the application effect of the modified CBE tool on pigs might not be very high, in the practice of single-base editing of porcine cells, different editing targets could be compared, and the targets with higher relative activity could be selected for application. For example, in this study the four candidate targets were analyzed and compared to select the most active target and more efficiently prepare CBE-mediated single-base editing cells of Bama minipigs, thereby laying the foundation for the next step of producing single-base edited pigs through somatic cell cloning.

In previous studies, MSTN-knockout was mainly achieved by CRISPR/Cas9-mediated random base insertion/deletion mutations leading to frameshift mutations (Li et al., 2020; Zhu et al., 2020b). Because the mutations are random and unpredictable, and CRISPR/Cas9 is prone to cause a series of adverse genomic consequences, there are many concerns about biosafety. This study was based on the characteristics of the directional conversion of a single $C \cdot G$ base pair to $T \cdot A$ base pair mediated by CBE through modification of the TGG codon corresponding to amino acid No. 203 of the MSTN coding box to a TAG, TGA or TAA stop codon. The translation of the MSTN mRNA to the stop codon produces a truncated MSTN protein without functional activity. This CBE-dependent design produced minimal changes to the genome. In addition, because the improved CBE had higher precision than the modified $\mathrm{CBE}$, the biosafety risk was lower. This strategy of introducing premature stop codons through the coding frame of the gene has been successfully utilized to construct knockout pigs. The editing of the porcine coat color gene, $T Y R$, is a successful example. Li et al. modified the CAG codon corresponding to amino acid No. 68 (glutamine) in TYR to a stop TAG codon by design, resulting in the termination of TYR mRNA translation at amino acid No. 68 and defective TYR protein function. This also caused albino symptoms in the geneedited pigs (Li et al., 2018).

In conclusion, this study constructed an "all-in-one" modified CBE plasmid that achieved the directional conversion of a single $\mathrm{C} \cdot \mathrm{G}$ base to $\mathrm{T} \cdot \mathrm{A}$ base pair in the editing window at multiple gene loci in cells of Bama minipigs. A base pair was used to prepare a single cell clone of Bama minipigs carrying MSTN prematurely termination, and this was shown to produce no detectable genome off-target effects. This study has provided a foundation for further application of somatic cell cloning to construct MSTN-edited Bama minipigs that only carry a single base mutation, thereby avoiding biosafety risks and providing a reference for the base editing of other genetic loci in Bama minipigs.

\section{Declarations}

\section{Acknowledgments}

Page $13 / 21$ 
This work was jointly supported by the Guangdong Provincial R\&D Project in Key Areas (2018B020203003), the National Natural Science Foundation of China (82070199), the Guangdong Basic and Applied Basic Research Fund (2019A1515110280), the Foshan Science and Technology Innovation Project (1920001001203) and the Guangdong Science and Technology Innovation Strategy Fund (The Special Fund for "Climbing Plan"; pdjh2020a0616) .

\section{Author Contributions Statement}

D.S.T., J.H.L. and X.X.Z. conceived and designed the experiments. J.S.P., Z.S.L., J.C.W. and X.X.Z. conducted the experiments and analyzed the results. J.F.G., X.H.W., Y.Y.L., W.J.L., Q.Y.L., Y.S.X., Y.R.C. and Y.H.C. participated in experiment assistant. J.S.P., D.S.T., J.H.L. and X.X.Z. drafted the manuscript. All authors participated in discussions of the results and reviewed the manuscript.

\section{Competing Interests}

All authors declare no competing financial and non-financial interests.

\section{References}

1. Anzalone AV, Koblan LW, Liu DR (2020) Genome editing with CRISPR-Cas nucleases, base editors, transposases and prime editors. Nat Biotechnol 38(7):824-844. https://doi.org/10.1038/s41587-020-0561-9

2. Burkard C, Lillico SG, Reid E, Jackson B, Mileham AJ, Ait-Ali T, Whitelaw CB, Archibald AL (2017) Precision engineering for PRRSV resistance in pigs: Macrophages from genome edited pigs lacking CD163 SRCR5 domain are fully resistant to both PRRSV genotypes while maintaining biological function. PLoS Pathog 13(2):e1006206. https://doi.org/10.1371/journal.ppat.1006206

3. Ding S-W, Xie Z, Pang D, Yuan H, Jiao H, Lu C, Wang K, Yang Q, Li M, Chen X, Yu T, Chen X, Dai Z, Peng Y, Tang X, Li Z, Wang T, Guo H, Li L, Tu C, Lai L, Ouyang H (2018) Genetically modified pigs are protected from classical swine fever virus. PLOS Pathogens 14(12):e1007193. https://doi.org/10.1371/journal.ppat.1007193

4. Doman JL, Raguram A, Newby GA, Liu DR (2020) Evaluation and minimization of Cas9-independent off-target DNA editing by cytosine base editors. Nat Biotechnol 38(5):620-628. https://doi.org/10.1038/s41587-0200414-6

5. Grobet L, Martin LJ, Poncelet D, Pirottin D, Brouwers B, Riquet J, Schoeberlein A, Dunner S, Ménissier F, Massabanda J, Fries R, Hanset R, Georges M (1997) A deletion in the bovine myostatin gene causes the doublemuscled phenotype in cattle. Nat Genet 17(1):71-74. https://doi.org/10.1038/ng0997-71

6. Haapaniemi E, Botla S, Persson J, Schmierer B, Taipale J (2018) CRISPR-Cas9 genome editing induces a p53mediated DNA damage response. Nat Med 24(7):927-930. https://doi.org/10.1038/s41591-018-0049-z

7. Han X, Gao Y, Li G, Xiong Y, Zhao C, Ruan J, Ma Y, Li X, Li C, Zhao S, Xie S (2020) Enhancing the antibacterial activities of sow milk via site-specific knock-in of a lactoferrin gene in pigs using CRISPR/Cas 9 technology. Cell Biosci 19(1):133. https://doi.org/10.1186/s13578-020-00496-y 10 ) .

8. He Z, Zhang T, Jiang L, Zhou M, Wu D, Mei J, Cheng Y (2018) Use of CRISPR/Cas9 technology efficiently targetted goat myostatin through zygotes microinjection resulting in double-muscled phenotype in goats. Biosci Rep 38(6):BSR20180742. https://doi.org/10.1042/BSR20180742

9. Ihry RJ, Worringer KA, Salick MR, Frias E, Ho D, Theriault K, Kommineni S, Chen J, Sondey M, Ye C, Randhawa R, Kulkarni T, Yang Z, McAllister G, Russ C, Reece-Hoyes J, Forrester W, Hoffman GR, Dolmetsch R, Kaykas A 
(2018) p53 inhibits CRISPR-Cas9 engineering in human pluripotent stem cells. Nat Med 24(7):939-946. https://doi.org/10.1038/s41591-018-0050-6

10. Jin S, Zong Y, Gao Q, Zhu ZX, Wang YP, Qin P, Liang CZ, Wang DW, Qiu JL, Zhang F, Gao CX (2019) Cytosine, but not adenine, base editors induce genome-wide off-target mutations in rice. Science 364(6437):292-295. https://doi.org/10.1126/science.aaw7166

11. Komor AC, Kim YB, Packer MS, Zuris JA, Liu DR (2016) Programmable editing of a target base in genomic DNA without double-stranded DNA cleavage. Nature 533(7603):420-424. https://doi.org/10.1038/nature17946

12. Kosicki M, Tomberg K, Bradley A (2018) Repair of double-strand breaks induced by CRISPR-Cas 9 leads to large deletions and complex rearrangements. Nat Biotechnol 36(8):765-771. https://doi.org/10.1038/nbt.4192

13. Li R, Zeng W, Ma M, Wei Z, Liu H, Liu X, Wang M, Shi X, Zeng J, Yang L, Mo D, Liu X, Chen Y, He Z (2020) Precise editing of myostatin signal peptide by CRISPR/Cas9 increases the muscle mass of Liang Guang Small Spotted pigs. Transgenic Res 29(1):149-163. https://doi.org/10.1007/s11248-020-00188-w

14. Li Z, Duan X, An X, Feng T, Li P, Li L, Liu J, Wu P, Pan D, Du X, Wu S (2018) Efficient RNA-guided base editing for disease modeling in pigs. Cell Discov 4:64. https://doi.org/10.1038/s41421-018-0065-7

15. Lv Q, Yuan L, Deng J, Chen M, Wang Y, Zeng J, Li Z, Lai L (2016) Efficient Generation of Myostatin Gene Mutated Rabbit by CRISPR/Cas9. Sci Rep 6:25029. https://doi.org/10.1038/srep25029

16. McPherron AC, Lee SJ (1997) Double muscling in cattle due to mutations in the myostatin gene. Proc Natl Acad Sci USA 94(23):12457-12461. https://doi.org/10.1073/pnas.94.23.12457

17. Petersen $B$ (2017) Basics of genome editing technology and its application in livestock species. Reprod Domest Anim 52(Suppl. 3):4-13. https://doi.org/10.1111/rda.13012

18. Qian L, Tang M, Yang J, Wang Q, Cai C, Jiang S, Li H, Jiang K, Gao P, Ma D, Chen Y, An X, Li K, Cui W (2015) Targeted mutations in myostatin by zinc-finger nucleases result in double-muscled phenotype in Meishan pigs. Sci Rep 5:14435. https://doi.org/10.1038/srep14435

19. Rao S, Fujimura T, Matsunari H, Sakuma T, Nakano K, Watanabe M, Asano Y, Kitagawa E, Yamamoto T, Nagashima $\mathrm{H}$ (2016) Efficient modification of the myostatin gene in porcine somatic cells and generation of knockout piglets. Mol Reprod Dev 83(1):61-70. https://doi.org/10.1002/mrd.22591

20. Ruan JX, Xu J, Chen-Tsai RY, Li K (2017) Genome editing in livestock: are we ready for a revolution in animal breeding industry? Transgenic Res 26:715-726. https://doi.org/10.1007/s11248-017-0049-7

21. Wang X, Niu Y, Zhou J, Yu H, Kou Q, Lei A, Zhao X, Yan H, Cai B, Shen Q, Zhou S, Zhu H, Zhou G, Niu W, Hua J, Jiang Y, Huang X, Ma B, Chen Y (2016) Multiplex gene editing via CRISPR/Cas9 exhibits desirable muscle hypertrophy without detectable off-target effects in sheep. Sci Rep 6:32271. https://doi.org/10.1038/srep32271

22. Wang Y, Bi D, Qin G, Song R, Yao J, Cao C, Zheng Q, Hou N, Wang Y, Zhao J (2020) Cytosine Base Editor (hA3ABE3-NG)-Mediated Multiple Gene Editing for Pyramid Breeding in Pigs. Front Genet 11:592623.

https://doi.org/10.3389/fgene.2020.592623

23. Wei YY, Zhan QM, Zhu XX, Yan AF, Feng J, Liu L, Li JH, Tang DS (2020) Efficient CRISPR/Cas9-mediated gene editing in Guangdong Small-ear Spotted pig cells using an optimized electrotransfection method. Biotechnol Lett 42(11):2091-2109. https://doi.org/10.1007/s10529-020-02930-0

24. Xie J, Ge W, Li N, Liu Q, Chen F, Yang X, Huang X, Ouyang Z, Zhang Q, Zhao Y, Liu Z, Gou S, Wu H, Lai C, Fan N, Jin Q, Shi H, Liang Y, Lan T, Quan L, Li X, Wang K, Lai L (2019) Efficient base editing for multiple genes and loci in pigs using base editors. Nat Commun 10(1):2852. https://doi.org/10.1038/s41467-019-10421-8 
25. Xu K, Zhou Y, Mu Y, Liu Z, Hou S, Xiong Y, Fang L, Ge C, Wei Y, Zhang X, Xu C, Che J, Fan Z, Xiang G, Guo J, Shang H, Li H, Xiao S, Li J, Li K (2020a) CD163 and pAPN double-knockout pigs are resistant to PRRSV and TGEV and exhibit decreased susceptibility to PDCoV while maintaining normal production performance. Elife 9:e57132. https://doi.org/10.7554/eLife.57132

26. Xu S, Kim J, Tang Q, Chen Q, Liu J, Xu Y, Fu X (2020b) CAS9 is a genome mutator by directly disrupting DNA-PK dependent DNA repair pathway. Protein Cell 11(5):352-365. https://doi.org/10.1007/s13238-020-00699-6

27. Yuan H, Yu T, Wang L, Yang L, Zhang Y, Liu H, Li M, Tang X, Liu Z, Li Z, Lu C, Chen X, Pang D, Ouyang H (2020) Efficient base editing by RNA-guided cytidine base editors (CBEs) in pigs. Cell Mol Life Sci 77(4):719-733. https://doi.org/10.1007/s00018-019-03205-2

28. Zhao JG, Lai LX, Ji WZ, Zhou Q (2019) Genome editing in large animals: current status and future prospects. Nat Sci Rev 6:402-420. https://doi.org/10.1093/nsr/nwz013

29. Zhu XX, Wei YY, Zhan QM, Yan AF, Feng J, Liu L, Lu SS, Tang DS (2020a) CRISPR/Cas9-mediated biallelic knockout of IRX3 reduces the production and survival of somatic cell-cloned Bama minipigs. Animals 10(3):501. https://doi.org/10.3390/ani10030501

30. Zhu XX, Zhan QM, Wei YY, Yan AF, Feng J, Liu L, Lu SS, Tang DS (2020b) CRISPR/Cas9-mediated MSTN disruption accelerates the growth of Chinese Bama pigs. Reprod Domest Anim 55(10):1314-1327. https://doi.org/10.1111/rda.13775

31. Zhu XX, Zhong YZ, Ge YW, Lu KH, Lu SS (2018) CRISPR/Cas9-mediated generation of Guangxi Bama minipigs harboring three mutations in a-synuclein causing Parkinson's disease. Sci Rep 8:12420. https://doi.org/10.1038/s41598-018-30436-3

32. Zuccaro MV, Xu J, Mitchell C, Marin D, Zimmerman R, Rana B, Weinstein E, King RT, Palmerola KL, Smith ME, Tsang SH, Goland R, Jasin M, Lobo R, Treff N, Egli D (2020) Allele-Specific Chromosome Removal after Cas9 Cleavage in Human Embryos. Cell 183(6):1650-1664.e15. https://doi.org/10.1016/j.cell.2020.10.025

33. Zuo E, Sun Y, Wei W, Yuan T, Ying W, Sun H, Yuan L, Steinmetz LM, Li Y, Yang H (2019) Cytosine base editor generates substantial off-target single-nucleotide variants in mouse embryos. Science 364(6437):289-292. https://doi.org/10.1126/science.aav9973

\section{Figures}


A

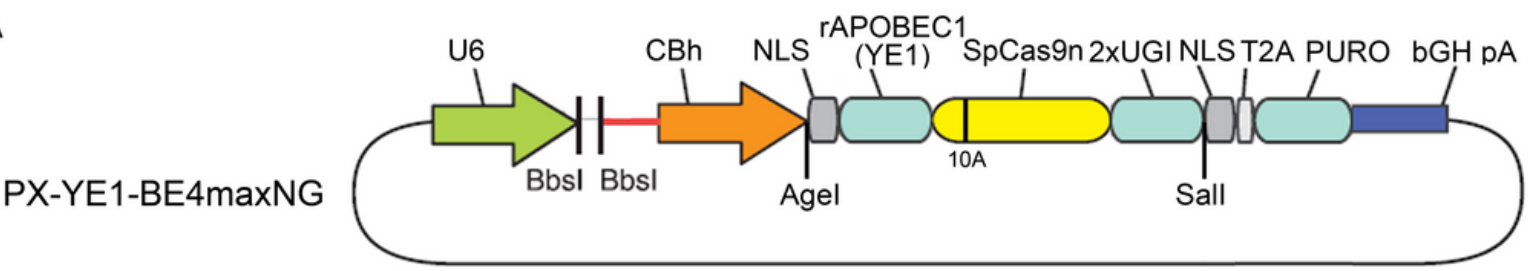

B

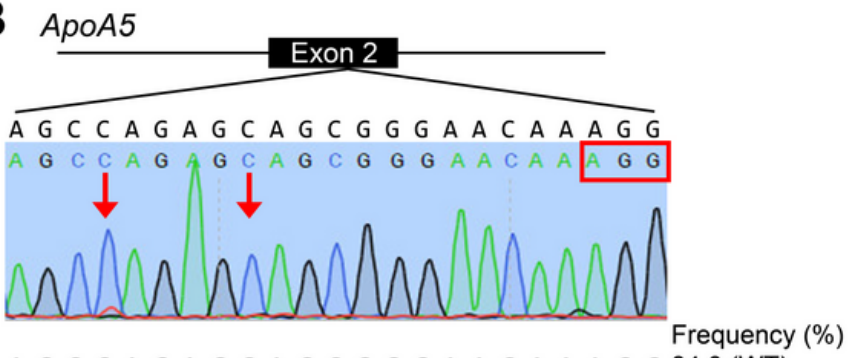

A G C C A G A G C A G C G G G A A C A A A G G 84.0 (WT)

A G C T A G A G C A G C G G G A A C A A A G G 7.4 (C-to- T)

A GCCA A G T A G C G G A A C A A A G 1.3 (C-to-T)

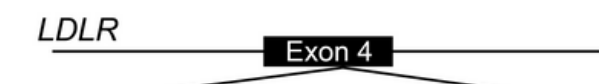

$\widehat{C C T G C A T C C C T G A G C T G T G G G C C}$

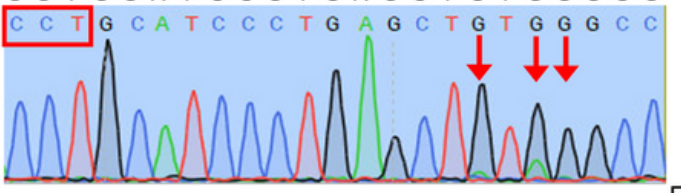

Frequency (\%)

C C T G C A T C C C T G A G C T G T G G G C C 60.7 (WT)

C C T G C A T C C C T G A G C T G T A G G C C 18.0 (C-to-T)

C C T G C A T C C C T G A G C T A T G G G C C 6.3 (C-to- T)

C C T G C A T C C C T G A G C T G T A A G C C 2.9 (C-to-T)

C C T G C A T C C C T G A G C T A T A G G C C 2.9 (C-to-T)

C

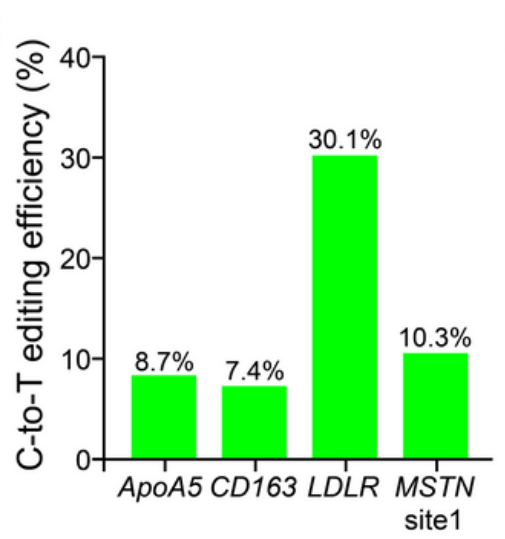

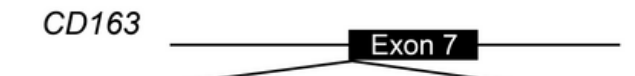
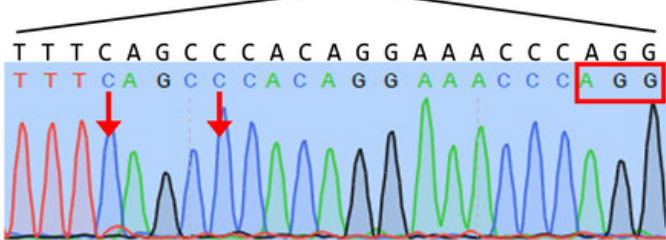

Frequency (\%)

T T T C A G C C C A C A G G A A A C C C A G G 88.4 (WT)

T T T TA GCC C A C A G G A A C C C A G G 6.5 (C-to-T)

T T T T A G C T C A C A G G A A C C C A G G 0.9 (C-to-T)

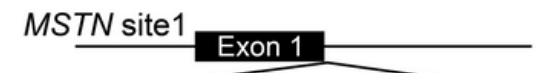

$\widehat{C C A G G A G A A G A T G G G C T G G T A A G}$

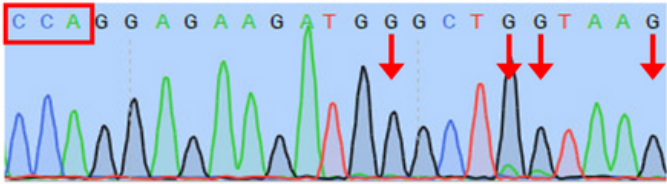

C C A G G A G A A G A T G G G C T G G T A A G 85.8 (WT)

C C A G G A G A A G A T G G G C T A A T A A G 6.1 (C-to-T)

C C A G G A G A G A T G G G T A G T A A G 1.8 (C-to-T)

C C A G G A G A A A T G G G C T A A T A A A 1.4 (C-to-T)

C C A G G A G A A G A T G G G C T G A T A A G 0.7 (C-to-T)

C C A G G A A A G T G A G C A A T A A G 0.3 (C-to-T)

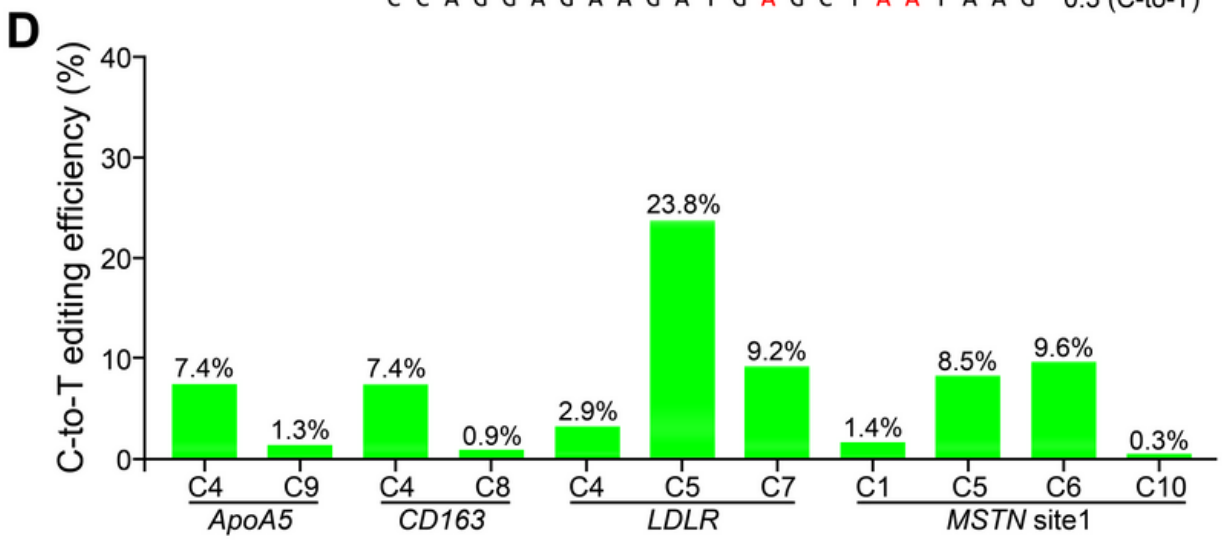

Figure 1

Activity test of the cytosine base editor (CBE) vector in cultured Bama minipig cells. (A) The constructed plasmid schematic view of "all-in-one" PX-YE1-BE4maxNG vector constructed in this study. The YE1-BE4maxNG sequence was synthesised and then linked them to the conventional PX459 vector. The expression of sgRNAs was initiated by an U6 promoter. The expression of CBE was controlled by CBh promoter. The PURO (puromycin) resistance gene was used for transfection screening in eukaryotic cells. These vectors could be linearized with Bbsl restriction enzyme for linking sgRNAs. (B) CBE-mediated C-to-T base-editing at four endogenous gene sites of Bama minipig cells. The gene sites in genome were indicated, the base-editing induced C-to-T (in the complementary chain is G-toA) conversions were indicated by red arrows, protospacer adjacent motif (PAM) sequences are labeled with red boxes. Representative C-to-T (in the complementary chain is G-to-A) base-editing induced hybrid peaks in Sanger 

conversion efficiency and positions at four tested genomic sites is presented in panels (C) and (D), respectively.

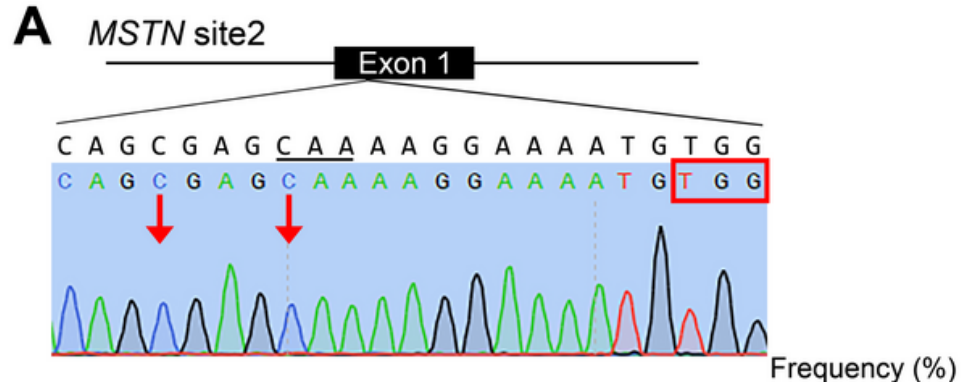

C A G C G A G C A A A A G G A A A T G T G G 96.0 (WT)

C A G C G A T A A A A G A A A T G T G G 1.0 (C-to-T)

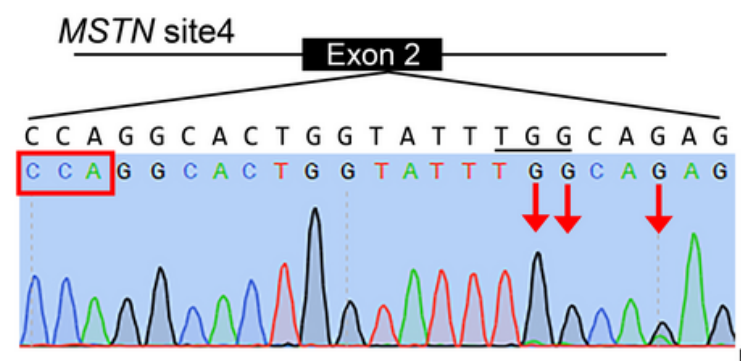

Frequency $(\%)$

C C A G G C A C T G G T A T T T G G C A G A G 64.8 (WT)

C C A G G C A C T G G T A T T T G G C A A A G 23.1 (C-to-T)

C C A G G C A C T G G T A T T T A A C A A G 5.6 (C-to-T)

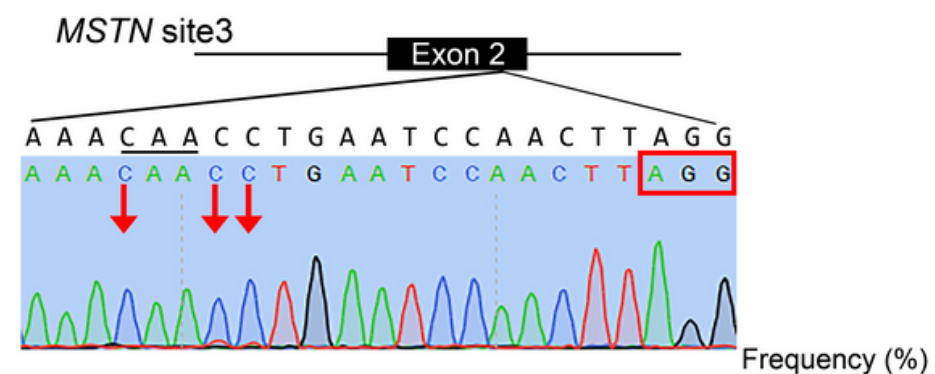

A A A C A A C C T G A A T C C A A C T T A G G 85.2 (WT)

A A A C A A T T T G A T C C A A C T T A G G 4.6 (C-to- $T$ )

A A A C A A T C T G A A T C C A A C T T A G G 3.8 (C-to-T)

A A A T A A C C T G A A T C C A A C T T A G G 1.1 (C-to-T)

A A A C A A C T T G A A T C A A C T T A G G 1.0 (C-to-T) MSTN site5

\section{Exon 2}
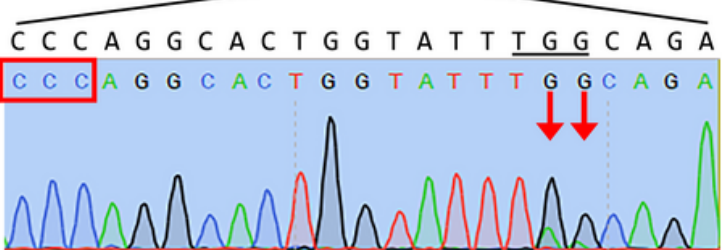

Frequency (\%)

C C C A G G C A C T G G T A T T T G G C A G A 66.3 (WT)

C C C A G G C A C T G G T A T T T A A C A G A 14.1 (C-to- T)

C C C A G G C A C T G G T A T T T A G C A G A 13.0 (C-to-T)

C C C A G G C A C T G G T A T T T G A C A G A 2.7 (C-to-T)
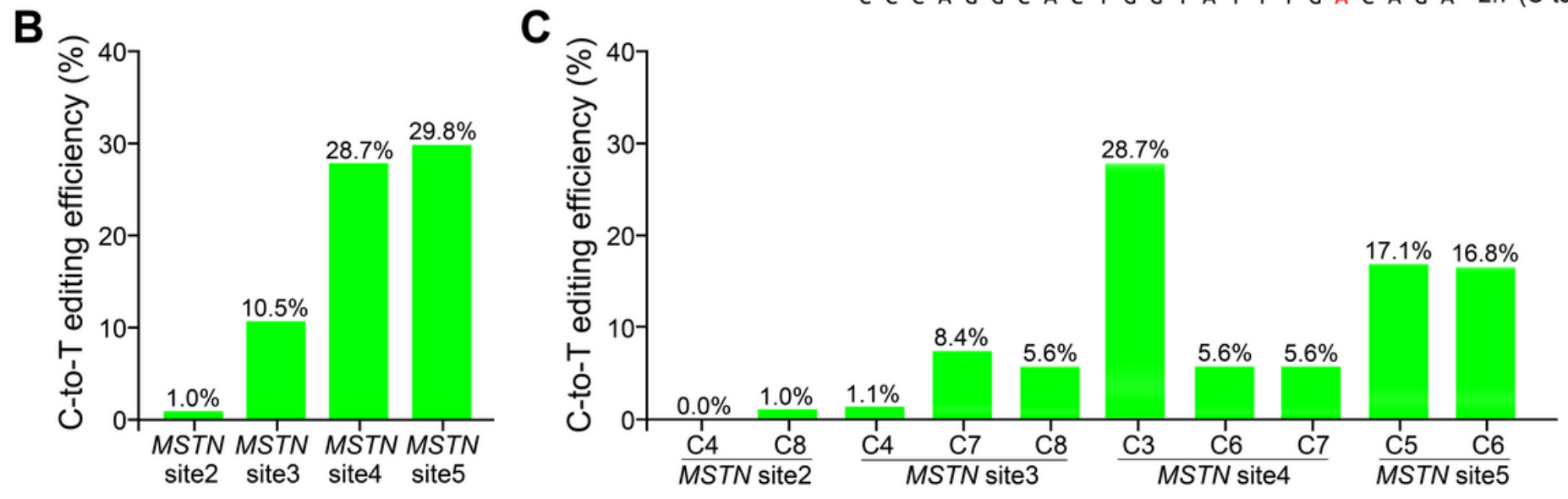

Figure 2

CBE-mediated base-editing in MSTN locus of Bama minipig cells. (A) Premature stop codons generated by CBEmediated C-to-T conversion were indicated unline in four targets from MSTN locus. The base-editing induced C-to-T (in the complementary chain is G-to-A) conversions were indicated by red arrows, protospacer adjacent motif (PAM) sequences are labeled with red boxes. Representative C-to-T (in the complementary chain is G-to-A) base-editing induced hybrid peaks in Sanger DNA sequencing results. Allele frequencies from deep sequencing are listed to the right. The CBE-mediated C-to-T conversion efficiency and positions at four tested genomic sites is presented in panels (B) and (C), respectively. 

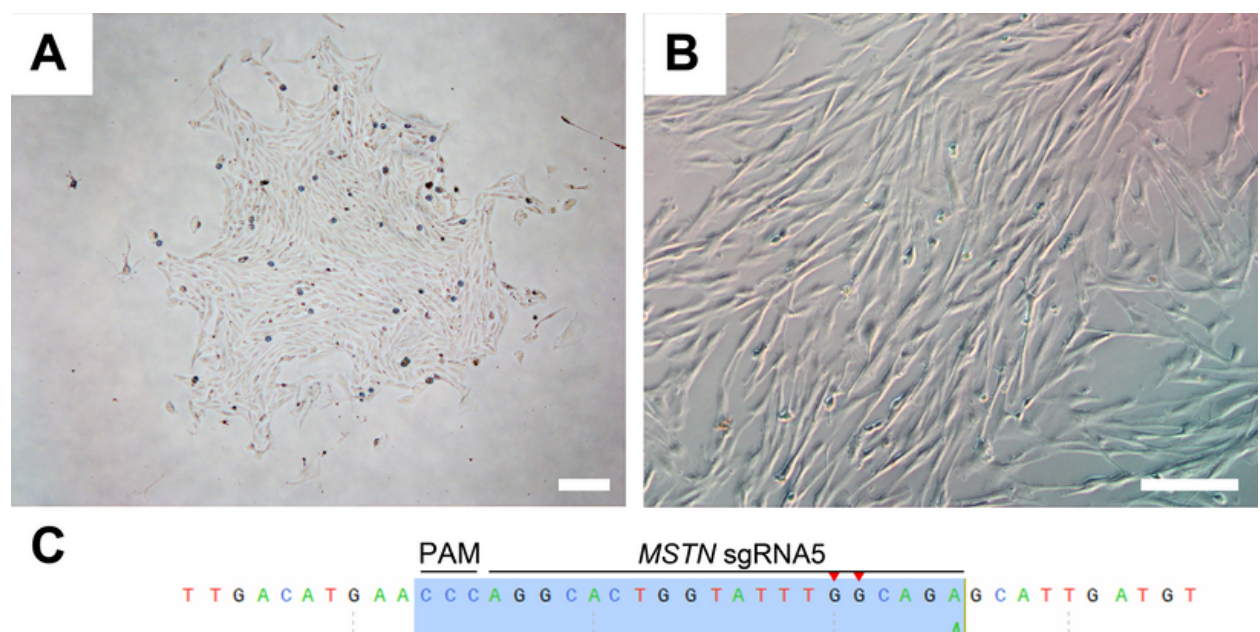

WT
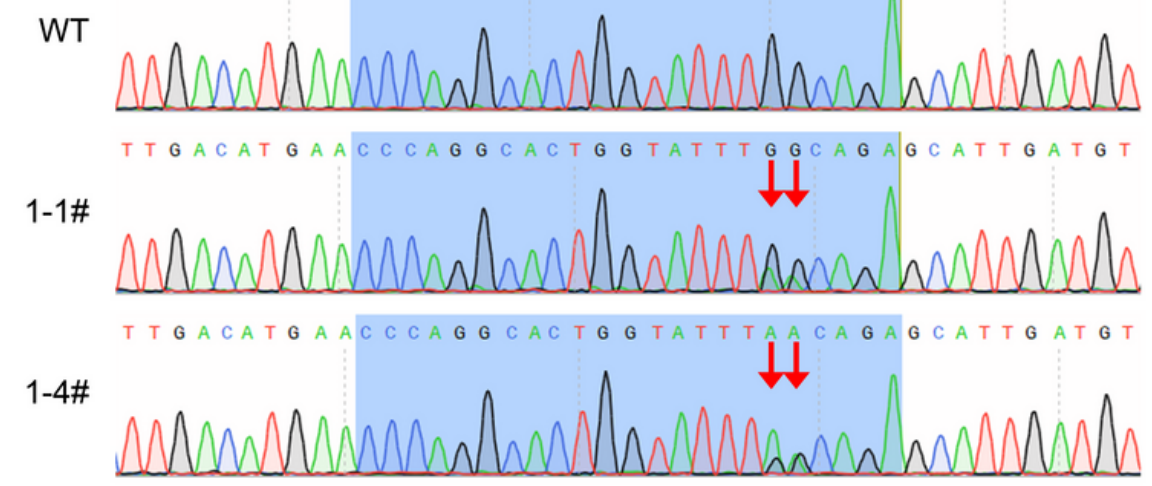

$1-6 \#$
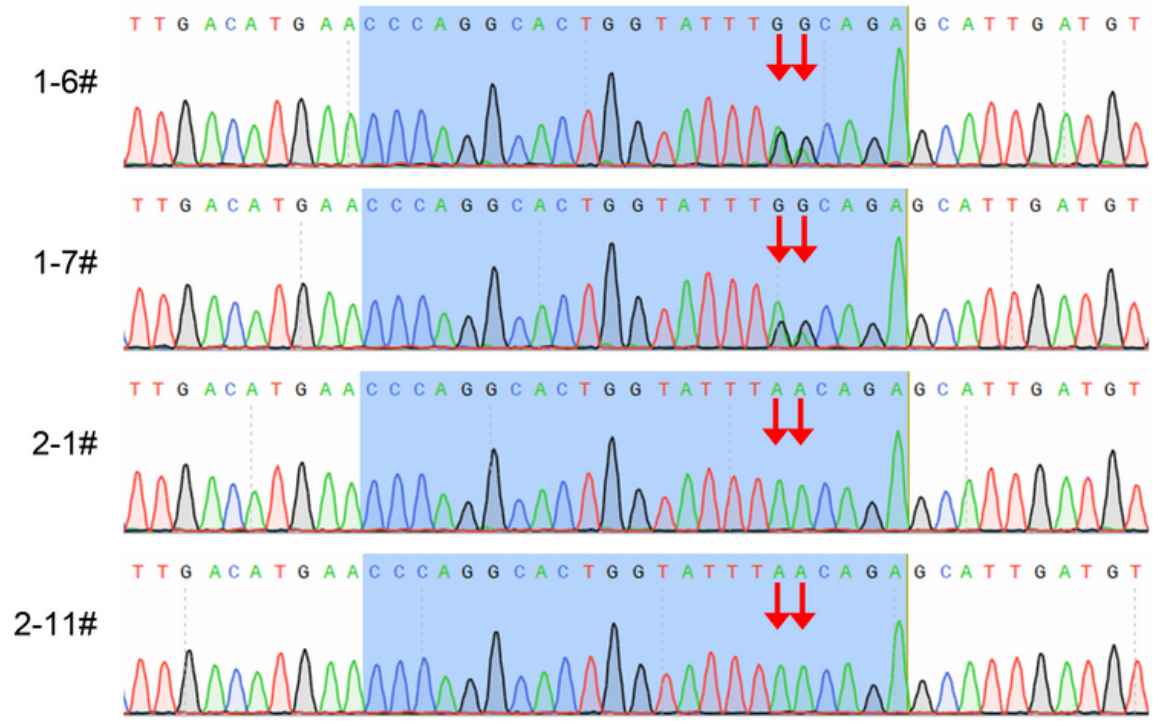

\section{Figure 3}

Preparation of Bama minipig cell colonies harboring CBE-mediated MSTN knock-out. (A) Representive single-cell colony emerged after cell transfection and puromycin screening. (B) Single-cell colonies were sub-cultured for 1 to 2 passages and showed good growth activity and cell morphology. (C) The base-editing target and protospacer adjacent motif (PAM) are indicating upon the wild type (WT) sequence. The base-editing induced C-to-T (in the complementary chain is G-to-A) conversions were indicated by red arrows. Sanger DNA sequencing results showed six single-cell colonies had the expected CBE-mediated C-to-T conversions (indicated by red arrows). Scale bars, 200 $\mu \mathrm{m}$. 
A
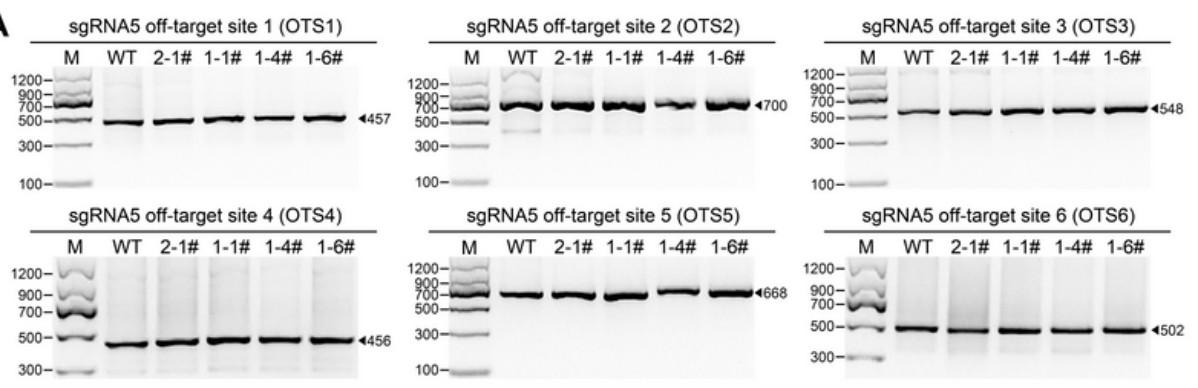

B
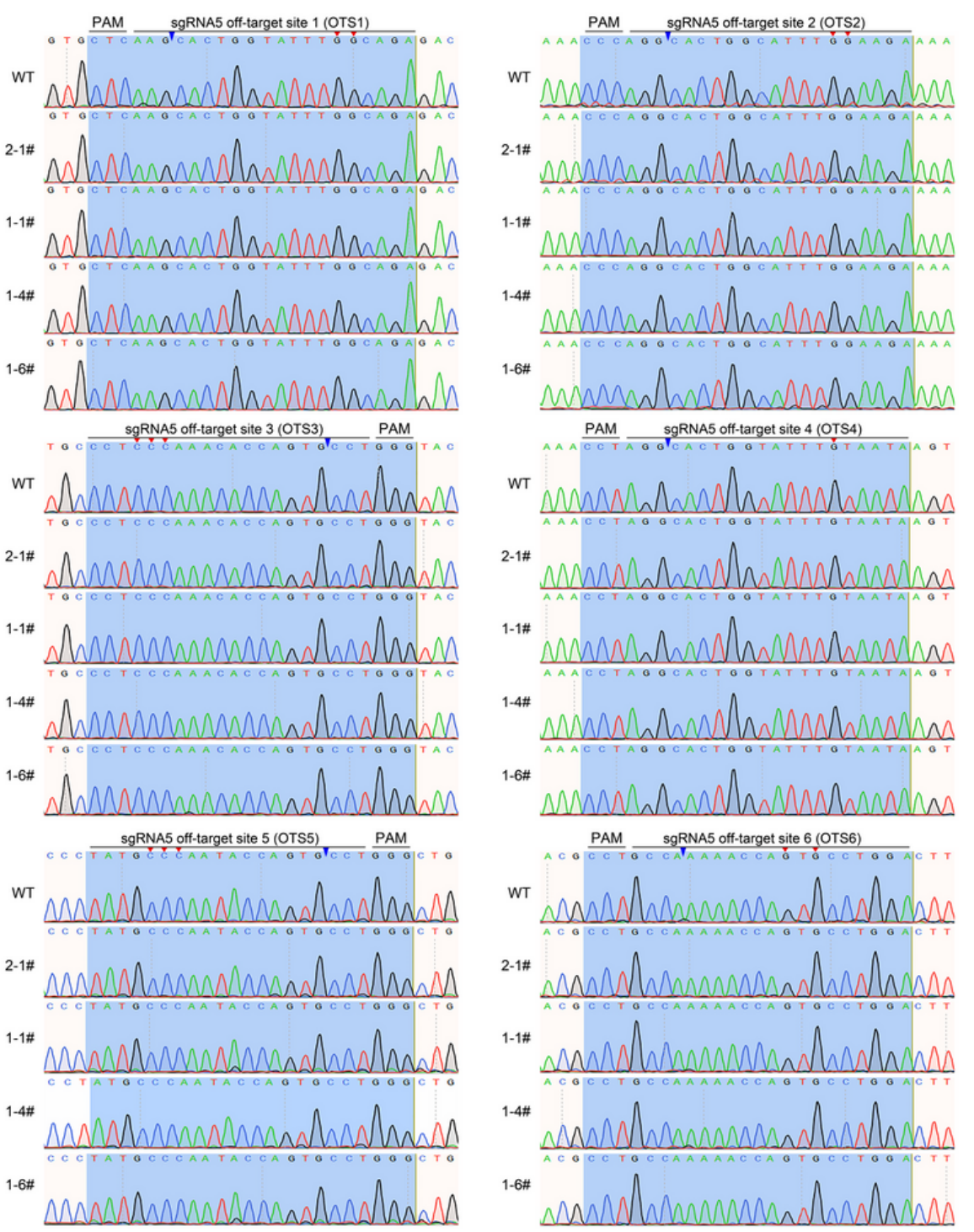

\section{Figure 4}

Detection of genomic off-target effects in CBE-edited Bama minipig cell colonies. Six genomic off-target sites with potential off-target effects were screened for off-target identification. Specific primers were used for PCR reaction, and the products $(A)$ were sequenced to confirm whether off-targeting mutations existed. The sample collected from wild type (WT) cells was used as a control. (B) Sanger DNA sequencing results are indicating no conversion of C.G base pairs to $T \cdot A$ base pairs at the six selected off-target sites (OTS) and no base deletion/insertion mutations caused by DNA double-strand breaks detected in the tested four CBE-edited single-cell colonies. The OTS targets and protospacer adjacent motif (PAM) are indicating upon the wild type (WT) sequences. The predicted base-editing induced C-to-T (in the complementary chain is G-to-A) conversions were indicated by red arrowheads. The funtional CRISPR/Cas9 induced DNA cleavage sites were indicated by blue arrowheads. 


\section{Supplementary Files}

This is a list of supplementary files associated with this preprint. Click to download.

- PXYE1BE4maxNG.dna 